\title{
Futuna. Un isolement pénalisant
}

\section{Marcel Gaillot}

\section{(2) OpenEdition}

Journals

Édition électronique

URL : http://journals.openedition.org/jso/6737

DOI : $10.4000 /$ jso. 6737

ISSN : $1760-7256$

Éditeur

Société des océanistes

Édition imprimée

Date de publication : 31 décembre 2012

Pagination : 265-268

ISBN : 978-2-85430-033-8

ISSN : 0300-953x

Référence électronique

Marcel Gaillot, «Futuna. Un isolement pénalisant », Journal de la Société des Océanistes [En ligne], 135 | 2012-2, mis en ligne le 27 novembre 2012, consulté le 25 septembre 2020. URL : http:// journals.openedition.org/jso/6737 ; DOI : https://doi.org/10.4000/jso.6737

(c) Tous droits réservés 


\title{
Futuna. Un isolement pénalisant ${ }^{1}$
}

\author{
par
}

Marcel GAILLOT*

L'isolement de Futuna, pendant les années 1942-1961 et même un peu au-delà, n'est pas toujours connu. Ce n'est que vers 1968 qu'une piste d'atterrissage lui ouvrit une porte sur le monde extérieur, favorisant ainsi la venue de certaines « élites » en mal de copie. Mieux, certains auteurs n'ont jamais mis les pieds à Futuna.

Le commandant de Brossard, pilote de Lancaster, effectuait une fois par mois une liaison avec Wallis. En 1962, il écrivait :

« S'il y a deux pistes d'avion à Wallis, Futuna et Alofi n'ont aucun terrain. [...] Chaque mois, [en effectuant] la liaison sur Wallis nous "droppons" un sac de courrier sur Futuna. Mais c'est une correspondance à sens unique. Pour les cas importants il n'y a que la radio. [...] Nous parlons en vieux camarades avec l'opérateur [le gendarme Perrot] à chaque voyage. Dès que nous avons le contact nous demandons d'abord les éléments météorologiques et enfin quelques phrases d'amitié volent vers cet homme dont nous ne connaissons que la voix déformée, portée par des ondes perturbées. »

Je précise que la zone de droppage en question se trouvait à $200 \mathrm{~m}$ d'altitude sur le plateau de Mauga, sur lequel il fallait tendre un drap blanc pour se signaler au pilote. L'accès à ce plateau par temps de pluie sur un sentier très pentu en terre argileuse n'était pas une sinécure. Ensuite, il fallait descendre à dos d'homme les sacs, car il n'y avait pas que le courrier mais aussi des quartiers de viande congelée. Il n'y avait donc guère de temps à perdre. ...

La période la plus éprouvante pour Futuna fut sans conteste les années 1942 à 1945 où l'île vécut dans le plus grand isolement pour cause de guerre dans le Pacifique après l'attaque par les Japonais de la base américaine de Pearl Harbor.

Dans sa chronologie de Wallis-et-Futuna (1963), le père O’Reilly souligne :

« [1945] Sans aucune liaison maritime, Futuna, sans farine sans sucre, sans remèdes et sans habits, connaît des jours difficiles. [...] Le 23 juin 1946, un sous-marin américain de passage à Futuna donna à la mission, sans ravitaillement depuis 2 ans, un peu de farine. »

C'est à la faveur de ce dénuement qu'un trader (collecteur de coprah) peu scrupuleux aurait profité de la situation pour abuser les Futuniens en échangeant des coupons de tissus stockés dans son magasin contre des parcelles de terrain plantées de cocotiers. Le tissu venant à manquer, il aurait continué son commerce en échangeant les sacs de jute servant d'emballage au coprah. Ce troc - car les Futuniens étaient totalement démunis d'argent - lui aurait rapporté une fortune considérable. En 1945, le résident de l'époque, M. Mattei, le mit dans l'obligation de restituer les biens acquis frauduleusement à leurs anciens propriétaires.

Cet isolement s'est poursuivi jusqu'à la fin des années 1960 où un aérodrome a été aménagé à Vele, seule plaine côtière pouvant accueillir ce projet au sacrifice d'une magnifique cocoteraie.

Antérieurement à la mise en place du statut de TOM, la période 1959-1961 fut passablement agitée. $\mathbf{M}^{\mathrm{gr}}$ Poncet (1972) relate la mise en place du référendum du 27 décembre 1959. C'est l'un des rares écrits disponibles sur cette période troublée. Il n'est pas inutile d'en rappeler l'essentiel :

1. NDLR. - Exceptionnellement, nous publions ici ce texte pensé par l'auteur comme un droit de réponse à Alisson Lotti mais qui pourra paraître à certains inachevé. Son auteur étant gravement malade, il n'a pu le reprendre et le corriger ; mais il lui tient à cœur de donner sa vision de cette histoire qu'il a vécue directement. Nous demandons donc aux lecteurs leur indulgence et leur compréhension.

* Gendarme à Futuna de septembre 1959 à mai 1961, chancelier de la Délégation, marcelgaillot@orange.fr 
«C'est le lundi 5 octobre [1959] que le ministre M. Soustelle arriva par un avion de la TAI [à Wallis]. On le voit, le général de Gaulle déléguait son ministre pour prendre contact avec les autorités du protectorat. [...] Comment fut préparée la demande faite au général de Gaulle, président de la République, par les trois rois de Wallis, Alo et Sigave, de faire des deux îles de Wallis et Futuna un "territoire d'Outre-mer de la République française" ? Il faut y voir la main du résident Fauché et du délégué de Futuna [Gloannec]. L'un et l'autre durent agir avec assez d'habileté pour faire aboutir le plan du haut-commissaire Péchoux en persuadant les trois rois autochtones des avantages très sérieux qu'en retirerait le pays. [...] Tout se passa cependant sans publicité. On apprit simplement un beau jour que la demande en question avait été faite. [...] »

Le dimanche 27 décembre 1959, l'ensemble de la population de Wallis-et-Futuna alla aux urnes pour la première fois. Il en ressortit un résultat stalinien à Wallis : unanimité à $100 \%$ ! À Futuna, les résultats furent un peu plus vraisemblables avec, pour le « oui », 84,50\% à Alo et $68,60 \%$ à Sigave.

Dans ce théâtre politique, $\mathrm{M}^{\mathrm{gr}}$ Poncet n'a vu que la scène sans pénétrer les coulisses. L'histoire de ce référendum reste à écrire et il faut espérer qu'elle le sera un jour. On a d'abord prétendu que les résultats de Futuna auraient été faussés à cause d'une phrase supprimée dans le décret $\mathrm{n}^{\circ}$ 59-1364 sur l'organisation du référendum, à savoir (article 9) :

« Si le résultat est négatif, les îles Wallis-et-Futuna conserveront leur statut actuel. »

Cela ressort d'une lettre adressée au député de Nouvelle-Calédonie, vraisemblablement au début de 1961 :

«Dans la traduction futunienne faite par le R.P. Simler pour le délégué du Résident, il n'a jamais été question de cette possibilité [de conserver le statut actuel même si le résultat est négatif]. Mieux, dans presque tous les villages, les gens ont voté "oui" sous la menace des chefs qui promettaient de fortes sanctions et qui ont obligé leurs populations à leur remettre les bulletins "non". Ceux-ci ont ensuite été présentés à l'administrateur-délégué. [...] Notre pays n'a jamais été aussi opprimé que depuis un an. Tout est maintenant motif à fortes amendes; les gens ne peuvent ni résider dans le village de leur choix, ni s'exprimer librement, ni voyager comme ils le veulent, ni travailler librement pour les maisons de commerce. Les lettres doivent être soumises à la censure du roi ; elles sont parfois confisquées pour être remises au Délégué. Tout cela pour des raisons de politique locale qui étaient inconnues jusqu'ici [...]. »

En octobre 1961 arrive à Wallis M. Périé, administrateur supérieur de la FOM en remplace- ment du résident Fauché. Il prend les contacts nécessaires avec les personnalités importantes et là, surprise ! C'est lui qui raconte (1994) :

«En fin d'après-midi, nous nous rendons avec Papilio [l'interprète officiel] à la maison du Lavelua (le roi), je n'ose pas dire le "palais royal", mais c'est en tout cas la seule maison en pierres de toute l'île, mis à part bien entendu la cathédrale et les églises de Mua et Lano [...]. Le Lavelua, d'une quarantaine d'années, se distingue par un air majestueux. Ils sont vêtus [le roi et ses six ministres] d'une chemise blanche, à manches longues, avec boutons de manchettes, flottant sur un petit pagne, le manu, qui laisse découvertes des jambes poilues. Le manu du roi est noir, celui des ministres rose ou vert. Après les salutations de politesse, le Lavelua aborde, par l'intermédiaire de l'interprète Papilio, la question qui lui tient à cœur, celle du statut. Il ne mâche pas ses mots et dit franchement qu'il n'en veut pas. Certes, lui et les gens d'Uvéa [Wallis] ont demandé à devenir français à part entière mais le hautcommissaire Péchoux et le résident leur ont affirmé que cela ne changerait rien à leurs coutumes et voilà maintenant qu'on parle de faire élire par tout le monde de nouveaux chefs. Ils n'en veulent pas, ni du député, ni du sénateur, ni des conseillers territoriaux. Ils veulent leurs coutumes, rien que leurs coutumes. Ils ont été trompés. Je lui réponds que c'est lui qui se trompe [...] Le Lavelua me dit être rassuré mais je ne suis pas certain qu'il le soit vraiment... et je pense qu'il n'aurait pas entièrement tort de ne pas l'être. [...]

Visite à $\mathrm{M}^{\mathrm{gr}}$ Poncet et à la mission mariste de Lano. $\mathrm{M}^{\mathrm{gr}}$ Poncet, un vieillard de quatre-vingts ans d'apparence, ne cache pas que c'est lui qui a poussé les trois rois d'Uvéa, d'Alo et de Sigave à demander à devenir "français à part entière" à une époque où de Gaulle décolonisait à tour de bras, mais il commence à douter d'avoir bien fait. »

Le dimanche 9 octobre 1961, M. Périé se rend à Futuna :

« J'ai appris par Gloannec que l'atmosphère politique était très tendue. Sous la pression de prêtres originaires du pays, le royaume d'Alo prétendant avoir été trompé sur le sens du référendum refusait le statut. Un groupe de chanteuses est allé jusqu'à chanter sur le thème : "On a été trompés, on veut l'indépendance" d'après la traduction de Papilio. »

M. Périé a été relevé de ses fonctions au bout de six mois, le 20 mars 1962. Catholique pratiquant, sa nomination avait visiblement au départ un arrière-goût éminemment politique : lever les doutes exprimés par $\mathrm{M}^{\mathrm{gr}}$ Poncet.

«Cette conséquence imprévisible de la campagne électorale fut immédiatement connue de toute l'île [...] L'évêque vint me dire [son] indignation et m'assurer de [son] soutien. J'ai vu $\mathrm{M}^{\mathrm{gr}}$ Poncet pleurer amèrement, regrettant d'avoir été à l'origine de la transformation du protectorat en territoire d'Outre-mer et ainsi d'avoir engagé les îles dans une évolution pleine de 
dangers pour leur personnalité et leur salut. [...] Ces événements concernant un petit territoire de Polynésie, ainsi qu'un modeste fonctionnaire, n'auraient que peu d'importance s'ils n'étaient le reflet de toute la politique d'outre-mer de la République, cinquième du nom, qui se révèle pire encore que les précédentes. Il est difficile d'accumuler autant de sottises politiques en aussi peu de temps que l'a fait la République dans les deux petites îles. Elle a perdu son prestige en trichant ouvertement à l'occasion des élections. Elle a combattu les chefs et notables qui étaient ses meilleurs alliés et soutenu contre eux ceux qui n'avaient cessé de la combattre. Elle a sérieusement inquiété la mission catholique qui se demande, à juste titre, si le but inavoué de l'Administration française n'est pas la lutte contre son influence et peut-être la déchristianisation des îles. »

Cette courte synthèse démontre s'il en était besoin que la situation dans cette période de 1959-1961 n'a pas été aussi idyllique que dépeinte par les médias. Elle fut émaillée d'incidents plus ou moins graves mais à l'échelle d'un petit pays comme Futuna cela prenait une importance énorme et maintenait sur les esprits une pression difficilement soutenable.

Un demi-siècle plus tard, ces incidents sont parfois relatés de façon fantaisiste voire mensongère par des personnes qui cependant font autorité en matière historique. Ainsi, récemment, prenant connaissance d'une excellente étude de M. Frédéric Angleviel (2006 : 61-76), quelle ne fut pas ma stupéfaction de découvrir page 71 le renvoi 27 reproduit ci-dessous :

«27. Allison Lotti donne le détail de la rixe du 31 juillet 1960 qui entraîna la mort de plusieurs Futuniens originaires d'Alo: "Il ne s'agissait pas d'un simple combat comme on en voyait régulièrement à la sortie de la messe, à mains nues sans armes. Un Futunien trouva un fusil et tira en l'air pour tenter d'arrêter la violence, les deux gendarmes réussirent sans trop de mal à le désarmer, et par la suite le délégué fit procéder à la confiscation de tous les fusils de l'île. Des hommes ne se relevèrent pas, ils furent transportés chez eux et moururent plusieurs jours après" (Lotti, 2005 : 46). »

Madame Lotti est docteur en histoire ; elle est la nièce de $\mathrm{M}$. Gloannec (le délégué de l'époque) ; elle avait donc la possibilité d'obtenir des éléments de première main tout en étant tenue de respecter la déontologie inhérente à sa charge. Je suis le dernier acteur vivant de cette période et je m'inscris en faux contre les affirmations relatées par $\mathbf{M}^{\mathrm{me}}$ Lotti. J'affirme que le vusu (bagarre à mains nues) s'est déroulé strictement entre gens de Sigave et qu'aucun Alo n'y a été mêlé de près ou de loin. De plus, il n'y a eu aucun mort mais seulement des blessés légers. Enfin, le Futunien en possession du fusil n'a jamais tiré « en l'air » pour la simple raison qu'il ne savait pas se servir d'un fusil à chien.

Je considère donc qu'il y a urgence à écrire l'histoire de cette période troublée, ô combien, à Futuna entre 1959 et 1961. Pour la mémoire des Futuniens de cette époque, j'espère qu'un historien qualifié et intègre entreprendra rapidement cette tâche avant qu'il ne soit trop tard.

En quelques lignes, je vais maintenant justifier mes affirmations. Un procès-verbal de gendarmerie dont je détiens un exemplaire a été établi et, à lui seul, fait justice des inexactitudes rapportées par $\mathrm{M}^{\text {me }}$ Lotti que nous allons reprendre ici.

Le Futunien qui « trouva un fusil » n'a jamais - fort heureusement - tiré un coup de feu pour la raison expliquée ci-dessus. Il s'agissait de Fulilagi Petelo dit «Aussia» de Nuku (Sigave) qui emprunta le fusil de chasse à un dénommé Sanualio (P.V. de gendarmerie du 31 juillet 1960 - Brigade de Futuna). À noter que ce Fulilagi Petelo a été choisi par le délégué lors d'un recrutement de jeunes susceptibles de faire des gradés dans l'armée. Il fit une carrière dans la gendarmerie. Il appartenait à la fraction favorable au délégué... Curieuse situation.

Autre inexactitude: "Les deux gendarmes réussirent sans trop de mal à le désarmer ». J'étais seul sur les lieux ; mon collègue Perrot n'y parvint qu'une demi-heure plus tard. J'étais donc seul pour désarmer Fulilagi dont l'arme était chargée ; il avait le doigt sur la détente.

L'affirmation que « Le délégué fit procéder à la confiscation de TOUS les fusils de l'île... » est encore fausse. Il ne s'agissait de saisir que les armes du royaume de Sigave puisque le royaume d'Alo n'a été mêlé ni de près ni de loin à ce vusu (pugilat à mains nues).

Enfin, «Des hommes ne se relevèrent pas, ils furent transportés chez eux et moururent plusieurs jours après » est le mensonge le plus grossier de la citation. Pour quelle motivation? Le médecin-lieutenant Lacoste a vu tous les blessés dont le plus grave présentait « une plaie de la région temporale droite, des fractures pluri costales, avec une incapacité de un mois sauf complications. » Un autre présentait une « fracture des os du nez». Les dix-neuf autres n'avaient que des " plaies minimes » ou des « contusions diverses " (P.V. du 31 juillet 1960 - Brigade de Futuna). Voici un court extrait de ce procèsverbal :

« Nous gendarme Gaillot, étant au domicile de Monsieur Michoutouchkine gérant de la S.C.I.E, avons entendu une rumeur venant de la rue en même temps qu'une foule assez importante arrivait à hauteur de la dite maison. Cette foule, scindée en deux groupes dont l'un semblait avoir l'avantage du nombre pro- 
gressait en direction du wharf de Leava. Les gens s'entrefrappaient à coups de poings, à coups de pieds et à coups de bâtons. [...] Quelques minutes plus tard, les gens semblèrent pris de panique et plusieurs voix s'élevèrent en même temps: "le tagata mo le fana" ("un homme avec un fusil"). En effet, un Futunien en position du tireur debout visait quelqu'un dans la foule. Nous nous élançâmes vers lui et, au moment où nous détournions le canon pour le désarmer, nous avons remarqué son doigt sur la détente... »

L'assertion selon laquelle Fululagi «tira en l'air pour arrêter la violence » ne tient pas. Au moment où je l'ai désarmé, son arme était bel et bien dirigée vers le groupe de Leava considéré comme rebelle envers le délégué... Ce qu'il confirme d'ailleurs dans son rapport. Un extrait du rapport $\mathrm{n}^{\circ}$ 4/4-Adm du 11 août 1960 de l'administrateur-délégué à Futuna sur la situation politique à Futuna et rixe du 31 juillet 1960, précise :

«Plus nombreux, les partisans du roi eurent vite le dessus et, de la place de l'église au magasin Lavoix, une vingtaine de leurs adversaires furent blessés à coups de poings. À ce moment-là, contrairement à la coutume qui veut que l'on se batte uniquement à mains nues, le jeune Petelo Fulilagi courut chercher un fusil dans la case d'Atou. Le gendarme Gaillot qui venait d'arriver sur les lieux parvint à le désarmer avant qu'il ne s'en serve. Interrogé plus tard [par qui ?], il prétendit avoir voulu simplement faire peur à ses adversaires sans intention homicide. [...] À quelques minutes de là, la population, surtout féminine, de Leava m'accueillit avec des cris et même des gestes hostiles. Le pugilat finissait alors. "

Les femmes n'étant pas admises dans les fono, elles s'expriment donc à leur manière.

On voit très bien qu'il est indispensable - la rigueur intellectuelle l'exige - que l'Histoire de cette île soit établie une fois pour toutes dans sa stricte exactitude par un (ou des) historien(s) à l'autorité incontestable et incontestée. Je suis à sa disposition autant que faire se pourra. Il n'existe pas, comme au temps des missions, de « diaire » relatant au jour le jour les événements importants, mais il subsiste des documents authentiques qui peuvent être utilement consultés avant qu'ils ne tombent dans l'oubli ou qu'ils ne disparaissent.

Dans les années 1950-1960, pratiquement aucun Futunien ne parlait, n'écrivait ni surtout ne lisait le français; ce n'est plus le cas aujourd'hui. La tradition orale quasi disparue ou en tout cas désuète ne facilite guère la pérennité et surtout l'exactitude des événements passés. Il importe de les figer une fois pour toutes, afin que les Futuniens puissent avoir accès à leur véritable Histoire.

Dans le cas de la citation de Madame Lotti, il eût été correct d'écrire :

« Il s'agissait en réalité d'un vusu (pugilat à mains nues) comme on en voyait quelquefois à la sortie de la messe. Sauf que cette fois, un jeune Futunien de Nuku (Sigave) trouva à se faire prêter un fusil mais ne sut s'en servir. Il fut désarmé par le gendarme sans qu'aucun coup de feu ne soit tiré. Il y eut 21 blessés dont un seul avec une incapacité d'un mois. Les vingt autres présentaient des blessures superficielles. Les protagonistes blessés étaient tous de Sigave : 10 de Leava, 10 de Nuku et un de Vaisei. »

Les faits auraient ainsi été relatés dans leur exactitude et il n'y aurait jamais eu de polémique sur ce sujet. L'adage «A beau mentir qui vient de loin » (Abbé Tuet, Matinées sénonaises ou Proverbes français - 1789) a pourtant encore de beaux jours devant lui...

\section{BIBLIOGRAPHIE}

ANGLEviEL Frédéric, 2006. Wallis-et-Futuna (19421961) ou comment le fait migratoire transforme le protectorat en том, Journal de la Société des Océanistes 122-123 : Spécial Wallis-et-Futuna, pp. 61-76.

Brossard Maurice (de), 1962. Tahiti, Océan des Français, Paris, Éd. France-Empire.

Lotтi Allison, 2005. Le passage du statut de Protectorat à celui de Territoire d'outre-mer à Wallis-etFutuna (1958-1962), DEA d'histoire, université de Bordeaux III, $152 \mathrm{p}$.

O’Reilly Patrick, 1963. Chronologie de Wallis-etFutuna, Journal de la Société des Océanistes 19, pp. 12-46.

PONCET Alexandre $\left(\mathrm{M}^{\mathrm{gr}}\right)$, 1972. Histoire de Wallis, T. II, Paris, Société des Océanistes, Publications de la SDO 23, pp. 224-227.

PÉRIÉ Jean, 1994. Administrateur des colonies, non repenti, Paris, La Pensée universelle. 